\title{
ARTICLE
}

\section{Fluence-to-dose conversion coefficients for aircrew dosimetry based on the new ICRP Recommendations}

\author{
Tatsuhiko SATO ${ }^{1, *}$, Akira ENDO ${ }^{1}$, Maria ZANKL ${ }^{2}$, Nina PETOUSSI-HENSS ${ }^{2}$, Hiroshi YASUDA $^{3}$ and \\ Koji NIITA $^{4}$ \\ ${ }^{1}$ Japan Atomic Energy Agency, 2-4, Shirakata-Shirane, Tokai, Ibaraki 319-1195, Japan \\ ${ }^{2}$ Helmholtz Zentrum München, German Research Center for Environmental Health (GmbH), Institute of Radiation \\ Protection, Ingolstaedter Landstraße 1, 85764 Neuherberg, Germany \\ ${ }^{3}$ National Institute of Radiological Sciences, 4-9-1, Anagawa, Inage, Chiba, 263-8555, Japan \\ ${ }^{4}$ Research Organization for Information Science and Technology, 2-4, Shirakata-Shirane, Tokai, Ibaraki 319-1106, \\ Japan
}

\begin{abstract}
The estimation of the fluence-to-effective dose conversion coefficients used especially for aircrew dosimetry is an important issue, since the geometrical conditions of aircrew exposure are considered not to be represented by the standard irradiation geometries such as the anterior-to-posterior (AP) and isotropic (ISO) geometries. We therefore calculated the dose conversion coefficients for neutrons and protons based on the ICRP 2007 Recommendations for three additional irradiation geometries: semi-isotropic irradiation from the upper hemisphere, and geometries closely representing the geometrical situations of aircrew exposure for two different flight conditions. The calculation was performed by the PHITS code coupled with the ICRP/ICRU adult reference computational phantoms. It was found from the calculation that (1) the conversion coefficients for the irradiation geometries closely representing the geometrical situations of aircrew exposure agree better with the corresponding data for the isotropic irradiation than those for the semi-isotropic irradiation; (2) the error associated with the assumption of the isotropic irradiation in the aircrew dosimetry is less than only $1 \%$ for conventional flight conditions. These findings indicate the adequacy of the use of dose conversion coefficients for the isotropic irradiation geometry in aircrew dosimetry.
\end{abstract}

\section{KEYWORDS: aircrew dosimetry, dose conversion coefficient, PHITS, voxel phantom, cosmic-ray}

\section{Introduction}

At high altitude, aircrews are subject to exposure to an increased level of cosmic radiation. During the last two decades, the radiation protection of aircrews has been intensively discussed all over the world, and as the results, many countries have issued regulations and recommendations setting an annual dose limitation for aircrews.

Recently, the ICRP released a new set of fundamental recommendations for a system of radiological protection in ICRP Publication 103 (ICRP103). ${ }^{1)}$ In this issue, the definition of effective dose was revised. Based on this revision, the fluence to the effective dose conversion coefficients for neutrons and protons up to $100 \mathrm{GeV}$ for the 6 irradiation geometries that were defined in ICRP Publication 74 (ICRP74) ${ }^{2)}$ were calculated using the PHITS code $^{3,4)}$ and the ICRP/ICRU adult reference computational phantoms. ${ }^{5)}$

However, the geometrical conditions of aircrew exposure were considered not to be represented by the standard irradiation geometries given in ICRP74, such as the anterior-to-posterior (AP) and isotropic (ISO) geometries. ${ }^{6}$ We therefore calculated the dose conversion coefficients for three additional irradiation geometries: semi-isotropic irradiation from the upper hemisphere, and

${ }^{*}$ Corresponding author, Tel. +81-29-282-5803, Fax.

+81-29-282-6063, E-mail: sato.tatsuhiko@jaea.go.jp geometries closely representing the geometrical situations of aircrew exposure for two different flight conditions. The latter geometries are referred to "aircrew-exposure geometries" in this paper. The angular distributions for the aircrew-exposure geometries were estimated from the PHITS simulation of the propagation of cosmic-rays in the atmosphere. ${ }^{7,8)}$ In order to examine the applicability of the operational quantity to aircrew dosimetry, the conversion coefficients for fluence to ambient dose equivalent $H^{*}(10)$ were also calculated.

The conversion coefficients for the standard irradiation geometries were given elsewhere.9) Hence, the results relevant to aircrew dosimetry will be shown in this paper, together with the analysis on aircrew doses obtained from different sets of dose conversion coefficients.

\section{Calculation Procedure}

Details of procedure for calculating the dose conversion coefficients using PHITS and the ICRP/ICRU adult reference computational phantoms have been reported in our previous paper. ${ }^{9)}$ Thus, the procedure only related to the source determination is described below.

Figure 1 illustrates the concept of the source determination in our simulation. The cosine of the zenith angle of each source particle, $\theta$, was randomly chosen from 0 to 1 for the semi-isotropic irradiation, while it was determined based on the angular distributions of the terrestrial cosmic-rays for the aircrew-exposure geometries. 


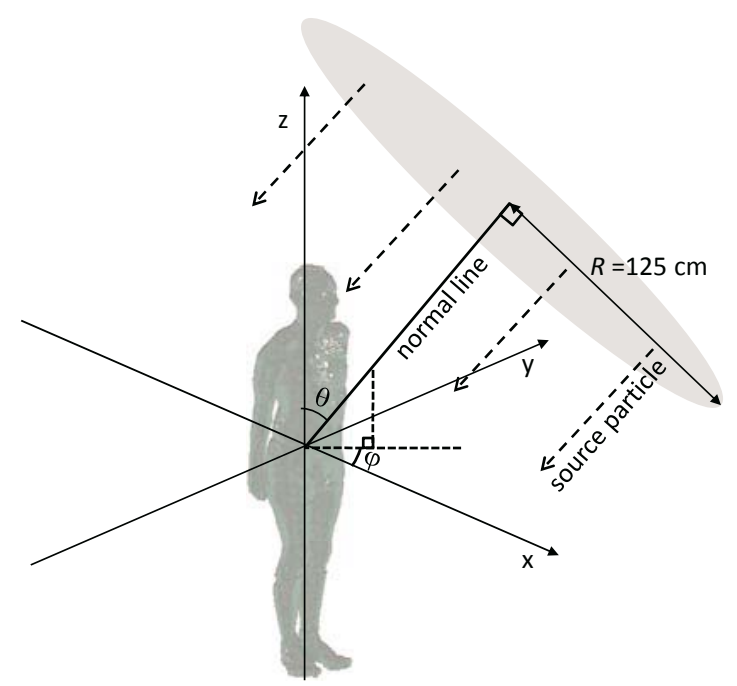

Fig.1 Illustration of the concept of the source determination in the simulation for calculating dose conversion coefficients

The azimuthal angle, $\varphi$, was randomly chosen from 0 to $2 \pi$ for both cases. The source position was determined by arbitrarily selecting a certain point from a disk with radius of $125 \mathrm{~cm}$ whose normal line across through its center is parallel to the source direction and toward to the origin of the system.

The angular distributions of the terrestrial cosmic-ray depend on the particle energy, the altitude, the force-field potential, and the vertical cut-off rigidity of the magnetosphere. The force-field potential is an index of the solar activity, and its numerical value can be determined from the count rates of several neutron monitors located all over the world. As examples, the neutron angular distributions at a conventional flight altitude are shown in Figure 2 for energies around $1 \mathrm{MeV}, 150 \mathrm{MeV}$ and $5 \mathrm{GeV}$ for two flight conditions: (1) near the magnetic pole during the solar minimum and (2) near the equator during the solar maximum. These two conditions are abbreviated to "P-min" and "E-max" in this paper. The vertical cut-off rigidities are 0 and $15 \mathrm{GV}$ for the P-min and E-max conditions, respectively, while their force-field potentials are 400 and $1200 \mathrm{MV}$, respectively. The data shown in the graph were estimated from the PHITS simulation of the propagation of cosmic-rays in the atmosphere. ${ }^{7,8)}$

It is evident from the graph that the angular distributions become closer to be isotropic with decreasing of the neutron energy. By comparing the angular distributions between the two flight conditions, it is found that their vertical directivities for the P-min condition are higher than the corresponding data for the E-max condition. In general, the vertical directivities of the angular distributions are higher for lower vertical cut-off rigidities and force-field potentials i.e. closer to the polar region and the solar minimum. Hence, the distributions for the two flight conditions shown in the graph are the highest and lowest vertical directivity cases at the altitude. From these

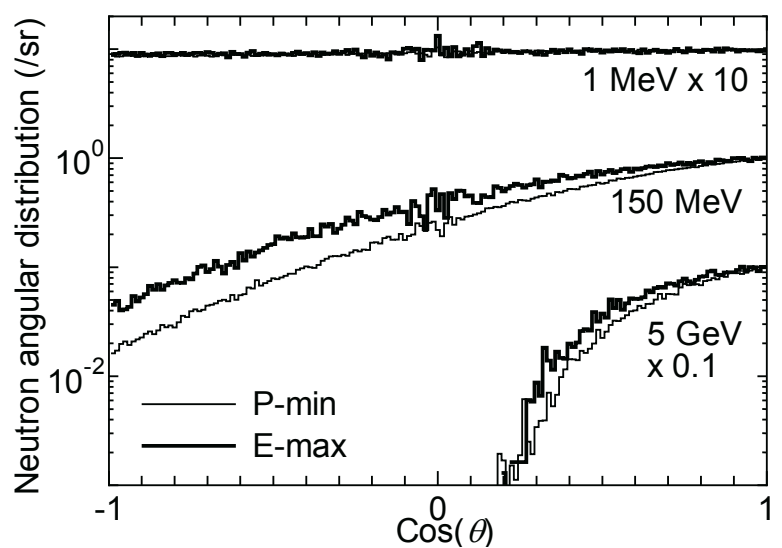

Fig.2 Neutron angular distributions calculated by PHITS at the altitude of $11 \mathrm{~km}$ for two flight conditions: near the magnetic pole during the solar minimum (P-min) and near the equator during the solar maximum (E-max). These data are normalized to 1 at the vertical direction.

considerations, we selected the angular distributions for the two flight conditions as extreme representatives of the aircrew-exposure geometries.

In order to calculate the dose conversion coefficients for these irradiation geometries, simulations were performed to estimate the absorbed doses in each organ of the reference phantoms, using the PHITS code. The fluence-to-effective dose conversion coefficients were then derived from the obtained organ doses and the radiation weighting factor, $w_{\mathrm{R}}$, and the tissue weighting factor, $w_{\mathrm{T}}$, following the instruction given in ICRP103.

\section{Results and Discussion}

\section{Fluence-to-dose conversion coefficient}

The calculated fluence-to-effective dose conversion coefficients for the semi-isotropic irradiation and the aircrew-exposure geometries are shown in Figure 3, in comparison with the corresponding data for the isotropic irradiation. ${ }^{9)}$ The fluence to $H^{*}(10)$ conversion coefficients calculated by PHITS are also plotted in the graphs. The statistical uncertainties of these data are generally small less than $2 \%$ in most cases.

It can be seen from the graphs that the conversion coefficients for the aircrew-exposure geometries agree better with the corresponding data for the isotropic irradiation rather than those for the semi-isotropic irradiation. Especially for neutrons with energy below 10 $\mathrm{MeV}$, the agreements between the data for the aircrew-exposure geometries and the isotropic irradiation are nearly perfect. This is because the angular distributions of such low energy neutrons are almost isotropic, as shown in Fig. 2, since those neutrons are generally produced by the evaporation process of nuclear reaction, which emits the particles in isotropic. On the other hand, the conversion coefficients for the semi-isotropic irradiation for the lower 

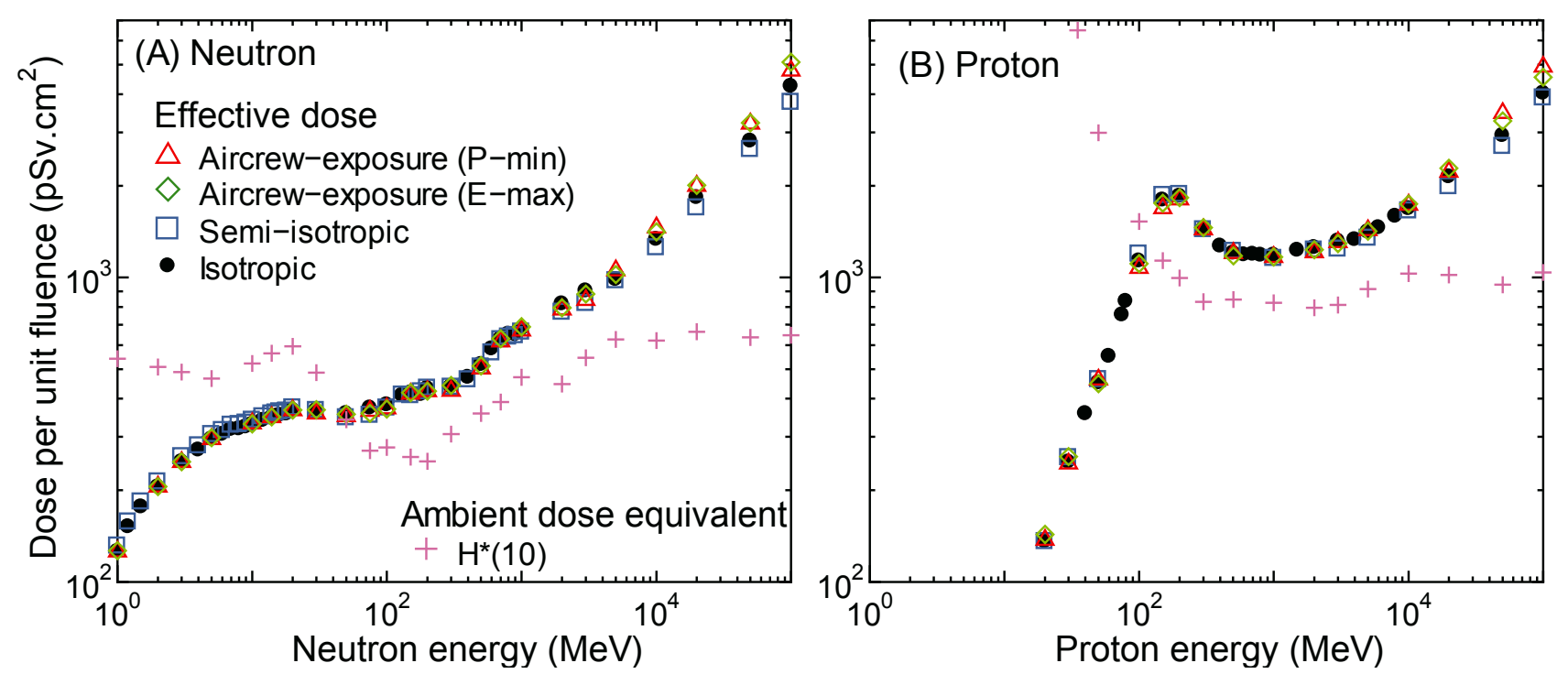

Fig.3 The fluence-to-effective-dose conversion coefficients for (A) neutrons and (B) protons calculated by PHITS and the ICRP/ICRU adult reference computational phantoms for various irradiation geometries. The fluence to $H^{*}(10)$ conversion coefficients calculated by PHITS are also plotted in the graphs.

energy neutrons slightly exceed the corresponding data for the other irradiation geometries, since the radiologically important organs or tissues such as breast and lung are concentrated in the upper side of the human body.

At energies over a few $\mathrm{GeV}$, the conversion coefficients for the aircrew-exposure geometries are larger than those for the isotropic irradiation, on the contrary to those for the semi-isotropic irradiation where the values are smaller than those for the isotropic irradiation. These tendencies can be explained by the fact that such high-energy particles can trigger a cascade of secondary particles by successively inducing complex nuclear reactions in the human body, and the absorbed doses inside the human body increase consistently with increasing distance from the incident surface for the high-energy irradiations. For the semi-isotropic irradiation and the aircrew-exposure geometries, the mean paths from the irradiation surface to the important organs are respectively shorter and longer than the corresponding values for the isotropic irradiation.

It is also found from Fig. 3 that the conversion coefficients for the two aircrew-exposure geometries agree with each other within their statistical uncertainties except for the incident energy over $10 \mathrm{GeV}$, where the contributions from such high-energy particles to the total aircrew doses are almost negligible. This result indicates that the difference of the angular distributions due to the variation of flight conditions hardly influences the aircrew doses.

The conversion coefficients for $H^{*}(10)$ are much greater than the corresponding data for the effective doses for neutrons with energy below $50 \mathrm{MeV}$ and protons with energy around $50 \mathrm{MeV}$, while the relations are reversed for other energies. These tendencies are predominantly attributed to the inconsistency between $10 \mathrm{~mm}$ and the mean paths from the incident surface to the important organs for the irradiation geometries. The influence of these discrepancies on the aircrew dose estimation will be discussed in the next section. Note that protons with energy approximately below $30 \mathrm{MeV}$ cannot reach to the $10 \mathrm{~mm}$ depth in the ICRU sphere, and thus, the dose conversion coefficients for $H^{*}(10)$ for such low energy protons are almost 0 .

\section{Aircrew dose estimation using the dose conversion coefficients}

The aircrew dose rates for the P-min and E-max conditions estimated using the obtained dose conversion coefficients are summarized in Table 1. The PHITS-based Analytical Radiation Model in the Atmosphere $(\text { PARMA })^{7,8)}$ was employed for calculating the terrestrial cosmic-ray spectra for those conditions. For comparison, the effective dose rates estimated based on the ICRP Publication 60 (ICRP60) $^{10)}$ are also presented in the table, where the dose conversion coefficients used in the estimation were calculated by PHITS coupled with the hermaphrodite MIRD-type phantom ${ }^{11)}$ using $w_{\mathrm{R}}$ and $w_{\mathrm{T}}$ as defined in ICRP60. ${ }^{12)}$

For both flight conditions, excellent agreements can be observed between the effective dose rates calculated using the dose conversion coefficients for the aircrew-exposure geometries and isotropic irradiation. These agreements verify that the error associated with the assumption of the isotropic irradiation in aircrew dosimetry is less than only $1 \%$ for most flight conditions at the conventional flight altitude. On the other hand, the dose rates calculated under the assumption of the semi-isotropic irradiation slightly exceed the corresponding data obtained using the dose 
Table 1 The aircrew dose rates in the unit of $\mu \mathrm{Sv} / \mathrm{h}$ for the P-min and E-max flight conditions estimated from the terrestrial cosmic-ray spectra calculated by PARMA multiplied with various sets of the dose conversion coefficients

\begin{tabular}{rcccccc}
\hline Condition for calculating & \multicolumn{2}{c}{ Neutron } & & \multicolumn{2}{c}{ Proton } \\
\cline { 2 - 3 } \cline { 6 - 7 } dose conversion coefficient & & P-min & E-max & & P-min & E-max \\
\hline \hline Aircrew-exposure (P-min) & 3.61 & 0.488 & & 1.46 & 0.207 \\
Aircrew-exposure (E-max) & 3.61 & 0.488 & & 1.48 & 0.209 \\
Semi-isotropic & 3.65 & 0.493 & & 1.49 & 0.207 \\
Isotropic & 3.62 & 0.489 & & 1.48 & 0.207 \\
Isotropic based on ICRP60 & 3.83 & 0.535 & & 3.30 & 0.474 \\
$H^{*}(10)$ & 5.05 & 0.673 & & 1.13 & 0.159 \\
\hline
\end{tabular}

conversion coefficients for the aircrew-exposure geometries. These results indicate that the aircrew doses can be precisely estimated under the assumption of the isotropic irradiation rather than the semi-isotropic irradiation. The use of the dose conversion coefficients obtained based on the definition of ICRP60 results in a slight increase of the effective doses for neutrons and a significant increase for protons. This tendency is predominantly attributed to the revision of $w_{\mathrm{R}}$ in ICRP103.

The calculated $H^{*}(10)$ for neutron are larger than the corresponding effective doses, although its conversion coefficients are much smaller than the data for the effective doses at high energies. This tendency indicates that the majority of neutron doses are attributed to the exposure of lower energy neutrons even in aircrew dosimetry. For example, approximately $56 \%$ of the total neutron doses are composed of the contribution from neutrons below $50 \mathrm{MeV}$. For proton, on the other hand, $H^{*}(10)$ are smaller than the corresponding effective doses. These findings show that $H^{*}(10)$ cannot be appropriately utilized as an operational quantity for proton dosimetry, since an operational quantity must exceed the respective protection quantity.

\section{Conclusions}

The fluence-to-effective dose conversion coefficients for neutrons and protons especially for aircrew dosimetry were calculated based on the ICRP 2007 Recommendations, utilizing the PHITS code and ICRP/ICRU adult reference computational phantoms. The following summarizes the conclusions derived from the calculation:

(1) The conversion coefficients for the irradiation geometries closely representing the geometrical situations of aircrew exposure agree better with the corresponding data for the isotropic irradiation rather than those for the semi-isotropic irradiation from the upper hemisphere. (2) The difference of the angular distributions due to the variation of flight conditions at conventional flight altitude hardly influences the aircrew doses. (3) The error associated with the assumption of the isotropic irradiation in aircrew dosimetry is less than only $1 \%$ for conventional flight conditions. (4) The revision in the ICRP 2007 Recommendation in terms of the definition of the effective dose results in a slight decrease of the effective doses for neutrons and a significant decrease for protons. (5) The ambient dose equivalent $H^{*}(10)$ cannot be appropriately utilized as an operational quantity for proton dosimetry.

Based on the first 3 facts, it was concluded that the dose conversion coefficients for the isotropic irradiation geometry are suitable to be used in aircrew dosimetry.

\section{Acknowledgement}

We would like to express our appreciation to the members of the ICRP Task Group on Dose Calculations (DOCAL) for their valuable comments and discussion. We also wish to thank the technical staff of the CCSE office of JAEA for their help in performing the Monte Carlo simulation.

\section{References}

1) ICRP, "The 2007 Recommendations of the International Commission on Radiological Protection," ICRP Publication 103 (2007).

2) ICRP, "Conversion coefficients for use in radiological protection against external radiation," ICRP Publication 74 (1996).

3) H. Iwase, K. Niita and T. Nakamura, "Development of a general purpose particle and heavy ion transport Monte Carlo code," J. Nuc. Sci. Technol. 39[11], 1142 (2002).

4) K. Niita, T. Sato, H. Iwase, et al., "Particle and heavy ion transport code system; PHITS," Radiat. Meas. 41, 1080 (2006).

5) ICRP, "Adult reference computational phantoms," ICRP Publication 110 (2009).

6) A. Ferrari and M. Pelliccioni, "On the conversion coefficients for cosmic ray dosimetry", Radiat. Prot. Dosim. 104, 211 (2003).

7) T. Sato and K. Niita, "Analytical function to predict cosmic-ray neutron spectrum in the atmosphere," Radiat. Res. 166, 544 (2006).

8) T. Sato, H. Yasuda, K. Niita, et al., "Development of PARMA: PHITS-based analytical radiation model in the atmosphere," Radiat. Res. 170, 244 (2008); http://phits.jaea.go.jp/expacs.

9) T. Sato, A. Endo, M. Zankl et al., Fluence-to-dose conversion coefficients for neutrons and protons calculated using the PHITS code and ICRP/ICRU adult reference computational phantoms," Phys. Med. Biol. 54, 1997 (2009).

10) ICRP, " 1990 Recommendations of the International Commission on Radiological Protection," ICRP Publication 60 (1991).

11) M. Cristy and K. F. Eckerman, Specific absorbed fractions of energy at various ages from internal photon sources. I. Methods, Oak Ridge National Laboratory Report ORNL/TM-8381/VI, (1987).

12) T. Sato, S. Tsuda, Y. Sakamoto, et al., "Analysis of dose-LET distribution in the human body irradiated by high energy hadrons," Radiat. Prot. Dosim. 106, 145 (2003). 